\title{
USO DEL CELULAR EN EL AMBIENTE LABORAL; RELACIÓN FEAR OF MISSING OUT - FOMO Y LOS ACCIDENTES DE TRABAJO
}

\author{
USE OF CELULAR IN THE WORK ENVIRONMENT; RELATIONSHIP BETWEEN FEAR OF MISSING \\ OUT - FOMO AND WORK ACCIDENTS
}

\author{
Juan Carlos Diaz Villamues \\ Uo801268@unimilitar.edu.co \\ Universidad Militar Nueva Granada \\ Administración de la Seguridad y Salud Ocupacional
}

Colombia

Resumen

La presente investigación, hizo énfasis en una problemática laboral con respecto al uso del celular, la relación del fenómeno Fear of Missing Out - FoMO y los accidentes de trabajo. En este sentido, los problemas que la adicción al celular ha generado en el entorno social, educativo y más específicamente en el laboral, demuestran un impacto negativo ya que los trabajadores por no usar controladamente el mismo, se distraen o disminuyen su capacidad de concentración, lo que termina por afectar esencialmente la salud e integridad física, y también, generar pérdidas significativas de productividad y dinero en las empresas.

Se presenta entonces a continuación un estudio cuantitativo, correlacional, con la cual se evidenció que el uso no controlado del celular en el trabajo aumenta significativamente las probabilidades de sufrir accidentes laborales.

Es una realidad que va en aumento, el celular se convierte progresivamente en el dispositivo tecnológico más utilizado en las empresas. La falta de atención y de concentración provocada por atender una llamada, responder un texto o ver la última publicación en la red social, son fuentes de accidentes o cuasi accidentes laborales y desafortunadamente algunos de esos accidentes han terminado en siniestros. En principio, existe un costo intangible que no se reflejan en cifras, y es el del sufrimiento humano ocasionado por los accidentes de trabajo, imposible estos de ser medidos. Por otro lado, se calcula que los días de trabajo perdidos por accidentalidad laboral representan cerca del 4 por ciento del PIB mundial y, en algunos países, hasta el 6 por ciento o más.

Ante el uso de los celulares por parte de los trabajadores, se plantean medidas en las empresas como el establecimiento de programas, normas y políticas internas de uso, creando conciencia hacia los trabajadores para así, disminuir los accidentes ocasionados por este distractor.

Palabras clave:

Accidentes, Trabajo, Celular, FoMO, Trabajador, Prevención. 


\section{INTRODUCCIÓN}

En los últimos tiempos, se ha ampliado la conciencia y la gravedad de los altos índices de accidentes laborales, así como de las muertes generadas por los mismos. A su vez que se toman medidas con un relativo éxito para la disminución de estas cifras. Ante la reducción de estos índices, es razonable también pensar ¿los accidentes laborales ya ocurridos hubieran sido evitables?, la mayoría de esos sucesos ¿no se relaciona con los descuidos o el bajo interés de las personas por la preservación de su integridad física?

En la actualidad, las tecnologías de la información y la comunicación se han integrado en la sociedad rápidamente, provocando un cambio trascendental en el estilo de vida y en la forma de interactuar, provocando cambios importantes, sobre todo en las actividades de ocio. En algunos estudios como el de Olivia (2007), se describe que el mayor riesgo del uso excesivo de las tecnologías es el de originar comportamientos adictivos que lleven no solo a una dedicación excesiva, sino a una verdadera dependencia y falta de control sobre las conductas (Sánchez-Torres, Arroyo-Cañada, Rojas-Berrio, Robayo-Pinzón, \& Fontalvo-Cerpa, 2019).

La consecuencia que más llama la atención es el FoMO (Fear of missing out), que se traduce en el miedo de perderse algún acontecimiento. Lo anterior significa ser un trastorno de ansiedad asociado al miedo de no estar al tanto de todo lo que está pasando. Este término está asociado principalmente al universo virtual, genera la necesidad de estar conectados permanentemente a Internet, consultando constantemente las redes sociales, el correo electrónico o aplicaciones de mensajería instantánea, y esto precisamente por el temor de no estar enterados de las cosas que suceden alrededor, o a quedar por fuera de algún evento o acontecimiento(Luis Martín; Trujillo-Flórez et al., 2019, 2018; Velandia Vargas et al., 2019).

Ahora, en el ámbito laboral, el uso de los celulares se ha convertido en una prioridad para la mayoría de los trabajadores, pues, así como son herramientas personales, también son equipos clave a la hora de trabajar y mantenerse en contacto con clientes, proveedores y entre los mismos compañeros de trabajo. Sin embargo, de no hacer un adecuado y controlado uso los celulares en el trabajo, desde la productividad, la seguridad y hasta la salud del trabajador pueden verse afectados; además de poner en riesgo los activos y los datos de la empresa (Niño-Benavides \& Cortés, 2018).

\section{Método}

Según el marco de investigación, se realiza una revisión bibliográfica utilizando un enfoque de carácter cuantitativo - correlacional, el cual permite relacionar por un lado el fenómeno FoMO y, por otro lado, los accidentes laborales. En consecuencia, esto deja a través del desarrollo investigativo, la asociación de las variables mediante un patrón predecible (Barba Abad Magdalena; Alvarado Nando, Maritza; De La Rosa Daza, Deisy; Mora Ramírez, Ángela Julieta, 2019; Daza-Orozco, 2019).

Dicho esto, en una primera fase se realizó la sistematización de la información consultada, su revisión y clasificación. Para así, estructurar el estado del arte y construir el marco teórico que se presentan en este trabajo como resultado del proyecto en curso. 
Gráfico método de investigación realizado

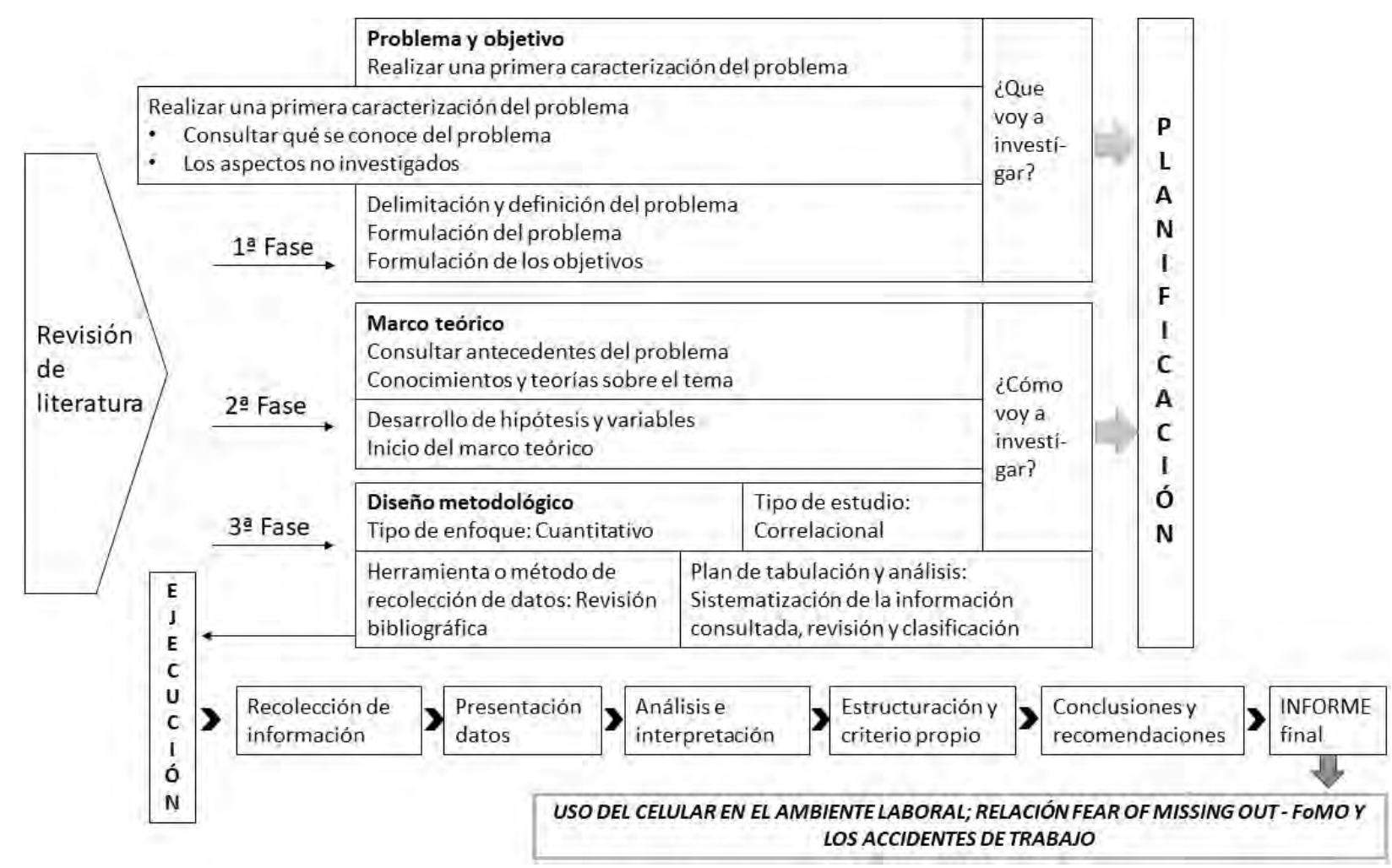

Gráfico 3 Método de investigación realizado. Elaboración propia

Por último, en cuanto a la especificación de la metodología relativa a una tradición epistemológica, tecnológica/disciplinar, se siguió la metodología disciplinar ya que se hizo búsqueda de información sobre dos temas principales y específicos, llevando a la compilación de dicha información para después llevarlo a un sistema ordenado y así llevar a cabo la investigación. Esta metodología disciplinar también estuvo acompañada de una investigación explicativa que se encargó de buscar el porqué de los sucesos mediante el establecimiento de la relación causa-efecto

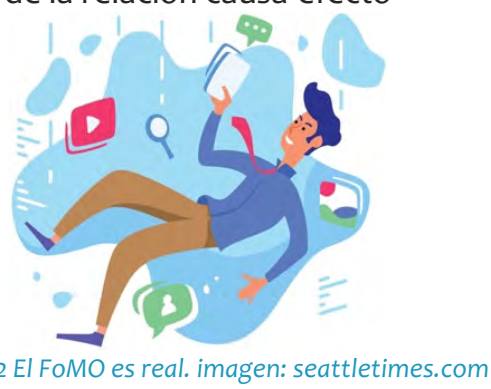

Resultados

El celular se convierte progresivamente en el dispositivo tecnológico más utilizado en las empresas, por encima de las laptops y las tabletas. 


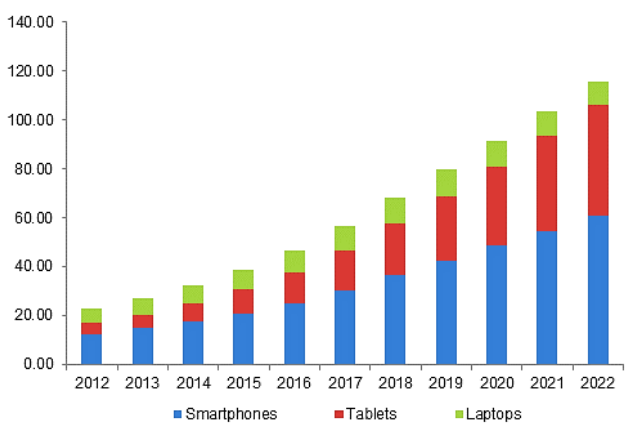

Imagen 2 Bring Your Own Device (BYOD) Market Size By End-Use (Small Businesses, Mid-To-Large Sized Businesses), By Device (Tablets, Smartphones, Laptops), Industry Analysis Report, Regional Outlook, Application Potential, Price Trend, Competitive Landscape \& Forecast, 2015 - 2022. Imagen: https://www.gminsights.com/

La falta de atención y de concentración provocada por atender una llamada, responder un texto o ver la última publicación en la red social, son fuentes de accidentes o cuasi accidentes laborales y desafortunadamente algunos de esos accidentes han terminado en siniestros (Norman-Acevedo, 2019).

- Se calcula que los días de trabajo perdidos representan cerca del 4 por ciento del PIB mundial y, en algunos países, hasta el 6 por ciento o más (Hämäläinen, Takala y Boon. 2017; Takala et al. 2014).

- El uso de los dispositivos móviles, específicamente los celulares, se incrementa exponencialmente a tal punto de generar perdidas en la productividad $y$, sobre todo, ser motivo de accidentes de trabajo. (Córdova, 2017).

- Al realizar una actividad, hace responsable del $25 \%$ de todos los accidentes a quien usa el aparato en horas de trabajo, ya que la actividad cerebral dedicada a la ejecución de las labores llega a disminuir hasta un 37\%, cuando se utiliza un teléfono celular”. (Córdoba, 2017)

- Así mismo, otros estudios aseguran que, el celular es revisado entre 80 y 110 veces por día y que el cerebro humano en promedio tarda 11 minutos en alcanzar el 100\% de la capacidad de concentración y 15 minutos en volver a recuperarla después de una interrupción.

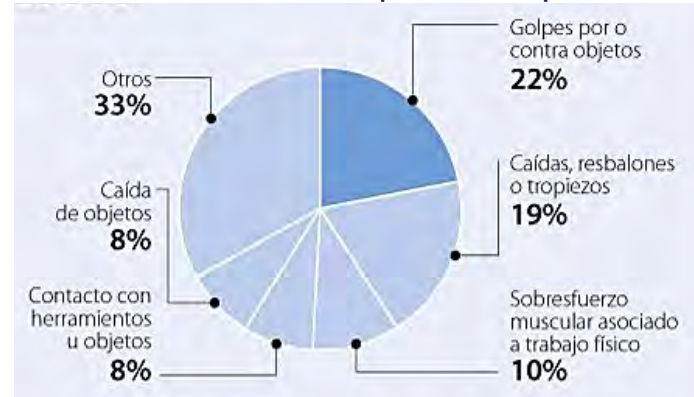

Imagen 3 Golpes y caídas son los accidentes laborales más comunes. Imagen: larepublica.co

- Es el mal manejo de las herramientas de trabajo y la falta de un procedimiento claro las principales causas de accidentes. También influye la falta de un ambiente de trabajo controlado y supervisado por un superior. Uno de los factores más comunes, según los expertos, es que los accidentados no prestan atención al protocolo del manejo de una herramienta, distrayéndose por usar el celular en el momento de la capacitación (SánchezTorres et al., 2019).

- Un estudio realizado por la multinacional de recursos humanos Adecco sobre el interés que le dan los colombianos al celular en el trabajo, refleja que el $86 \%$ de los participantes cuanta 
con un celular Smartphone. Así mismo, el 44\% de los encuestados afirma que no revisan su celular frecuentemente en el trabajo, seguido de un $26 \%$ que declara revisarlo cada hora.

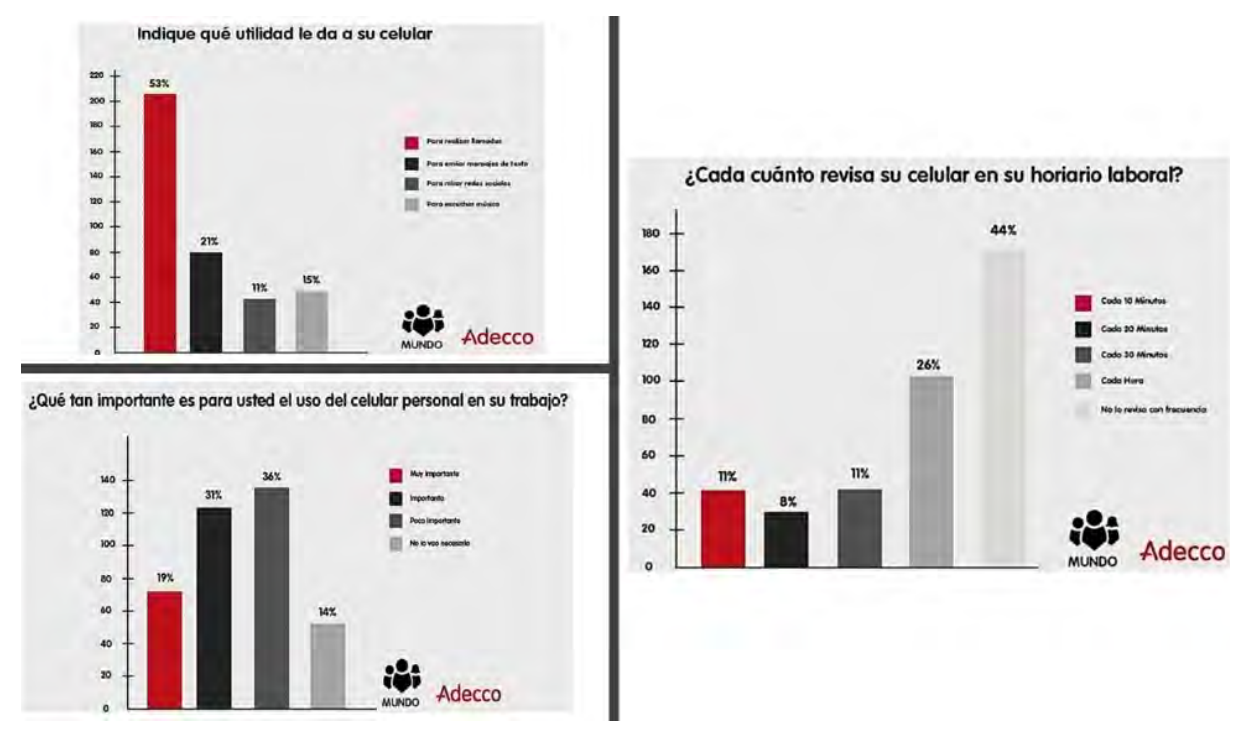

Imagen 4 ¿Afecta el uso del celular la productividad laboral? Imagen: adecco.com.co

- En el ambiente laboral, en las reuniones de trabajo, es normal que todos pongan sus celulares sobre la mesa casi como armas preparadas para ser usadas en cualquier momento. Así pues, Laos (2018) comenta: "Más allá de escribir o grabar mensajes por WhatsApp o de interactuar por Facebook o Twitter, el celular parece estar diseñado para que experimentemos sensaciones distintas a las que habíamos sentido con cualquier pantalla anterior".

\section{Discusión y Conclusión}

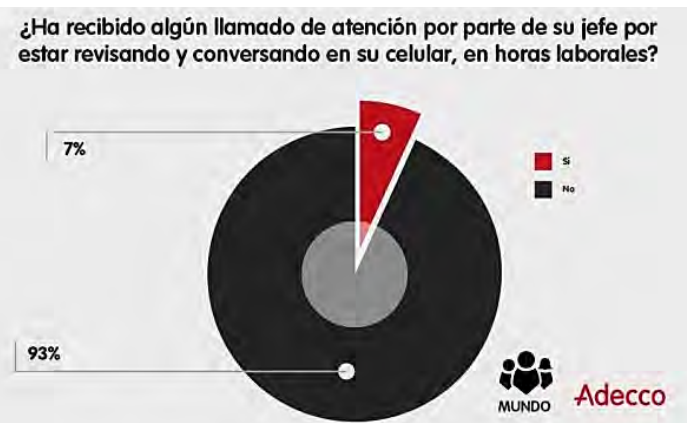

Imagen 5 Llamados de atención de parte de los empleadores por el uso del celular en horarios

laborales. Imagen: adecco.com.co

Generalmente se refiere la no prohibición de los teléfonos celulares toda vez que, a esta altura de la evolución tecnológica, el uso de estos pueda llegar a ser considerado un derecho esencial del trabajador, en situaciones en las cuales existan razones de riesgo o sea necesario lograr un nivel de atención extremo, el empleador tiene la posibilidad de limitar el uso de los teléfonos celulares en diversos espacios de la empresa o en determinados horarios. (Estudiogrispo, 2017). Esto, acompañado de una comunicación precisa por parte del empleador con pautas claras respecto a las conductas esperadas en la empresa en relación con la utilización razonable de los celulares en horarios laborales-

También, ante el uso de celulares por parte de los trabajadores se deben plantear seriamente medidas en las empresas como establecer normas o políticas de uso, para así disminuir los accidentes generados por la distracción del mismo. Todo esto importante ya que tendrá el fin de: 
- Dar a conocer a los trabajadores lo que está permitido dentro del horario laboral (en cuanto a acción, forma, tiempo y lugar).

- Esclarecer y establecer los derechos y obligaciones de los trabajadores dentro de las empresas.

- Entender el porqué de las restricciones, y tomar las decisiones de actuación, con pleno conocimiento de las consecuencias en caso del incumplimiento.

- Proteger a todos los miembros de la empresa de los riesgos del uso no controlado de los celulares.

- Encontrar un equilibrio que permita la convivencia positiva y productiva entre los miembros de la organización y las tecnologías de comunicación.

- Contribuir ampliamente a la Visión, Misión y Objetivos de la empresa

En cuanto a la cultura de la prevención, además de la importancia de una política relacionada al uso de los celulares y pese a que durante el 2018 se redujeron estos incidentes, en comparación al año anterior, es elemental que los trabajadores y en sí las empresas, realmente acojan una cultura de prevención laboral, una cultura del autocuidado, todo esto para reducir significativamente el número de accidentes y siniestros relacionados con el trabajo.

Es por esto por lo que, desde el año 2003 cada 28 de abril se celebra el Día Mundial de la Seguridad y la Salud en el Trabajo, instituido por la Organización Internacional del Trabajo (OIT). Un día el cual busca crear conciencia, sensibilizando a los trabajadores y las empresas de la importancia de la prevención de los riesgos laborales, para garantizar ampliamente la tranquilidad, la protección y en general, la calidad de vida de los trabajadores.

El llamado es a reforzar los lazos entre todos los actores del sistema laboral para lograr una real cultura de la prevención. Un trabajador sin conciencia de la prevención de riesgos se encuentra aún más propenso a sufrir accidentes laborales(Ramírez, 2011).

Aunque en materia de reducción de accidentes laborales ha habido avances, las cifras siguen siendo inquietantes, y más preocupante es que los sucesos en este ramo de los seguros (ARL) alcanza el 60 por ciento, En otras palabras, significaría que por cada 100 pesos que ingresaron a las ARL, por motivos de siniestros en accidentes salieron 60 pesos asignados a cubrir los costos de las accidentalidades laborales.

Igualmente, en Colombia solo en el año 2017, por ese concepto de accidentalidad laboral, las aseguradoras tuvieron que destinar más de 1,3 billones de pesos, lo que significa un 12,1\% por encima los datos dados en 2016, esto según cifras de la Superintendencia Financiera.

\section{Referencias bibliográficas}

Addeco. ¿Afecta el uso del celular la productividad laboral? [Sede Web]. Colombia: Adecco; 2017- [Actualizada el 14 de abril de 2017; acceso el 8 de julio de 2019]. Disponible en: $w w w$. adecco.com.co

Barba Abad, M; Alvarado Nando, M; De La Rosa-Daza, D; Mora-Ramírez, ÁJ. (2019). Innovación educativa, nuevas metodologías y experiencias en el aula, experiencias desde México y Colombia. Bogotá: Institución Universitaria Politécnico Grancolombiano.

Bhutani, A., \& Bhardwaj, P. (2016) Bring Your Own Device (BYOD) Market Size By End-Use, By Device, Industry Analysis Report, Regional Outlook, Application Potential, Price Trend, Competitive Landscape \& Forecast, 2015 - 2022. [Gráfico]. Recuperado de https://www.gminsights.com/industry-analysis/bring-your-own-device-BYOD-market

Braff, D. (2019). FOMO stress is real - even for adults. [Figura]. Recuperado de https://www.seattletimes.com/explore/shopnorthwest/fomo-stress-is-real-even-for-adults/

Córdoba, G. Uso de celulares en los centros de Trabajo. Cero accidentes. ISSN 2017 - 8412. 2016; Volumen 5: 1-12

Estudio Grispo. El uso del celular durante la jornada laboral [Sede Web]. Argentina: Estudio Grispo; 2017- [Actualizada el 19 de mayo de 2017; acceso 4 de julio de 2019]. Disponible en: www.estudiogrispo.com.ar

Daza-Orozco, CE. (2019). Iniciación científica: conceptualización, metodologías y buenas prácticas. Bogotá: Sociedad Colombiana de Investigación e Innovación Formativa - SCOIF. Retrieved from http://www.scoif.com/iniciacioncientifical 
Giraldo, J. Golpes y caídas son los accidentes laborales más comunes [Sede Web]. Colombia: La República; 2018- [Actualizada el 13 de junio de 2018; acceso 7 de julio de 2019]. Disponible en: $\underline{w w w . l a r e p u b l i c a . c o}$

Hämäläinen, P.; Takala, J.; Boon Kiat, T. 2017. Global Estimates of Occupational Accidents and Workrelated Illnesses 2017 (XXI Congreso Mundial de Seguridad y Salud en el Trabajo, Singapur, Workplace Safety and Health Institute).

Laos, J. La adicción al celular, un problema de humanidad [Sede Web]. Colombia: El Tiempo; 2018- [actualizada el 22 de septiembre de 2018; acceso 9 de julio de 2019]. Disponible en: www.eltiempo.com

Niño-Benavides, T. D. P., \& Cortés, M. I. C. (2018). Strategic communication and corporate social responsibility scenarios and potentialities in the creation of social capital: A review of literature. Prisma Social, (22), 128-158. Retrieved from https://www.scopus.com/inward/record.uri?eid=2-s2.0-

85054977237\&partnerID=40\&md5=b8cb6a62e42ccdef1d994274d7132b4b

Norman-Acevedo, E. (2019). Consumer cultural studies. Bogotá: Institución Universitaria Politécnico Grancolombiano.

Olivia. (2017). Síndrome del fomo en los estudiantes de la institución educativa secundaria "josé carlos mariátegui" aplicación de la una - puno en el año escolar 2017. Universidad Nacional del Altiplano, 1, p.11.

Ramírez, A. C. (2011). Attitudes of micro and small business entrepreneurs towards the acquisition of external information for making business decisions . Estudios Gerenciales, 27(121), 159-173. https://doi.org/10.1016/S0123-5923(11)70186-3

Sánchez-Torres, J. A., Arroyo-Cañada, F. J., Rojas-Berrio, S. P., Robayo-Pinzón, O. J., \& Fontalvo-Cerpa, W. (2019). The Colombian electronic consumer: Analysis of the leading factors of e-commerce use. International Journal of Electronic Marketing and Retailing, 10(3), 283-308. https://doi.org/10.1504/IJEMR.2019.100704

Trujillo-Flórez, LM, Bernal Yermanos, M. Á., Escobar Castro, J. A., González Triana, M. C., Gutiérrez Carvajal, J. P., Gutiérrez Vanegas, J. C., ... Torres López, A. L. (2019). Experiencias de innovación educativa - Tomo 3 (1st ed.; Luis Martín TrujilloFlórez, ed.). Bogotá: Politécnico Grancolombiano.

Trujillo-Flórez, LM, Martínez Contreras, R. M., Espitia López, H., Rojas Paredes, J. C., Vargas Leguizamón, Y. R., \& Castro Cabal, G. A. (2018). Experiencias de innovación educativa (1st ed.; L. M. Flórez-Trujillo, ed.). Bogotá: Politécnico Grancolombiano.

Velandia Vargas, G. K., Malangón Torres, M. A., Ramos Duarte, N., Quiroz Rubiano, M. M., Rodríguez Rubio, A., \& Celemín Pabón, Y. A. (2019). Experiencias de innovación educativa - Tomo 2 (1st ed.; Luis Martín Trujillo-Flórez, ed.). Bogotá: Politécnico Grancolombiano. 